\title{
Reaction Between 7-Hydroxy Coumarin, Alkyl Isocyanides and Dialkyl Acetylenedicarboxylate: Synthesis of $\mathbf{4 H}$-Chromenes and 1-Azabuta-1,3-dienes
}

\author{
Bita Mohtat, ${ }^{1, *}$ Saeedeh Farsijani, ${ }^{1}$ Maryam Razaghi, ${ }^{1}$ and Hoorieh Djahaniani ${ }^{2}$ \\ ${ }^{1}$ Department of Chemistry, Karaj Branch, Islamic Azad University, Karaj, Iran. b_mohtat@yahoo.com \\ 2 Department of Chemistry, East Tehran Branch, Islamic Azad University, Qiamdasht, Tehran, Iran
}

Received January 12, 2013; accepted April 11, 2013

\begin{abstract}
The reactive intermediate generated by the addition of tertbutyl and 1,1,3,3-tetramethyl butyl isocyanide to dialkyl acetylenedicarboxylate was trapped by 7-hydroxycoumarin to produce highly functionalized $4 H$-chromenes in fairly good yields. When the reaction is performed with cyclohexyl isocyanide, 1-azabuta-1,3-dienes were obtained.

Key words: 7-hydroxycoumarin, dialkyl acetylenedicarboxylates, alkyl isocyanides, multicomponent reactions.
\end{abstract}

\section{Introduction}

In recent years, isocyanide-based multicomponent condensation reactions (IMCRs) by virtue of their synthetic potential, their inherent atom efficiency, convergent nature, ease of implementation, and the generation of molecular diversity, have attracted much attention because of the advantages that they offer to the field of combinatorial chemistry [1,2]. The fact that complex products can be formed in a single operation by simultaneous reactions of several reagents has caused IMCRs to be among the most powerful methods for the synthesis of organic molecules [3]. It has been shown that alkyl or aryl isocyanides add to dialkyl acetylenedicarboxylates to generate zwitterionic species, which serve as intermediates in many different reactions [4-9].

The reactions of 1-azadienes with various chiral dienophiles, leads to substituted pyridines [10], and substituted $4 \mathrm{H}$ chromenes are a new class of anti-cancer compounds [11]. 2-Amino- $4 H$-chromenes have been of interest because of their biological activity [12] and some methods have been reported for their synthesis [13-20]. As part of our current studies [21$26]$ on the development of new routes to heterocyclic systems, we now report an efficient synthetic route to 2-amino- $4 \mathrm{H}$ chromenes (4) using alkyl isocyanides (1), 7-hydroxycoumarin (2) and alkyl acetylenedicarboxylate (3) (Scheme 1). When the reaction was performed with cyclohexyl isocyanide, 1-azabuta1,3-dienes (5) were obtained (Scheme 2).

\section{Result and Discussion}

The reaction proceeded spontaneously in $\mathrm{CH}_{2} \mathrm{Cl}_{2}$, and was completed within a few hours. The ${ }^{1} \mathrm{H}$ - and ${ }^{13} \mathrm{C}$-NMR spectra of the crude products clearly indicated the formation of $\mathbf{4}$ and 5 . The structures of compounds $\mathbf{4}$ and $\mathbf{5}$ were deduced from their
Resumen. Los intermediarios reactivos generados por la adición de isocianuros de t-butilo y 1,1,3,3-tetrametilbutilo a acetilendicarboxilatos de dialquilo fueron atrapados por 7-hidroxicumarina para producir $4 \mathrm{H}$-cromenos altamente funcionalizados en buenos rendimientos. Cuando las reacciones se llevan a cabo con isocianuro de ciclohexilo, se obtuvieron 1-azabuta-1,3-dienos.

Palabras clave: 7-hidroxicumarina, acetilendicarboxilatos de dialquilo, isocianuros de alquilo, reacciones de multi-componentes.

elemental analyses and their IR, ${ }^{1} \mathrm{H}-\mathrm{NMR}$ and ${ }^{13} \mathrm{C}-\mathrm{NMR}$ spectra. The mass spectra of these compounds displayed molecular ion peaks at appropriate $\mathrm{m} / \mathrm{z}$ values. The NH proton resonance at $\delta=8.76$ disappeared after addition of $\mathrm{D}_{2} \mathrm{O}$ to the $\mathrm{CDCl}_{3}$ solution of $4 \mathbf{a}$. The proton decoupled ${ }^{13} \mathrm{C}$ NMR spectrum of $4 \mathbf{a}$ showed the presence of oxo and amino groups at one end of the double bond leads to polarization of the olefinic system. The $\alpha$ carbon atom of this polarized system appears at $\delta=172.9$ while and the $\beta$-carbon at $\delta=72.1 \mathrm{ppm}$. Similar chemical shifts have been observed for the polarized carbon-carbon double bonds in 2-alkylamino-4H-benzo[h]chromene derivatives [13].

The ${ }^{1} \mathrm{H}$ NMR spectrum of 5a exhibited three sharp lines for methoxy ( $\delta=3.72$ and $3.86 \mathrm{ppm})$, and methine $(\delta=6.17$ ppm) protons. The cyclohexyl and coumarin moiety appeared at $\delta=1.20-2.07$ and $\delta=6.63-7.80 \mathrm{ppm}$. The ${ }^{13} \mathrm{C}$ NMR spectrum of 5a showed distinct resonances in agreement with the proposed structure.

NMR spectroscopy was employed to distinguish between $(Z)-5$ or $(E)-\mathbf{5}$. The $E$ configuration of the olefinic double bond in $\mathbf{5 a - b}$ is based on the chemical shift of the olefinic proton [27]. The ${ }^{1} \mathrm{H}$ NMR spectra of (E)-5 showed the olefinic proton signal at $7.08-7.10 \mathrm{ppm}$.

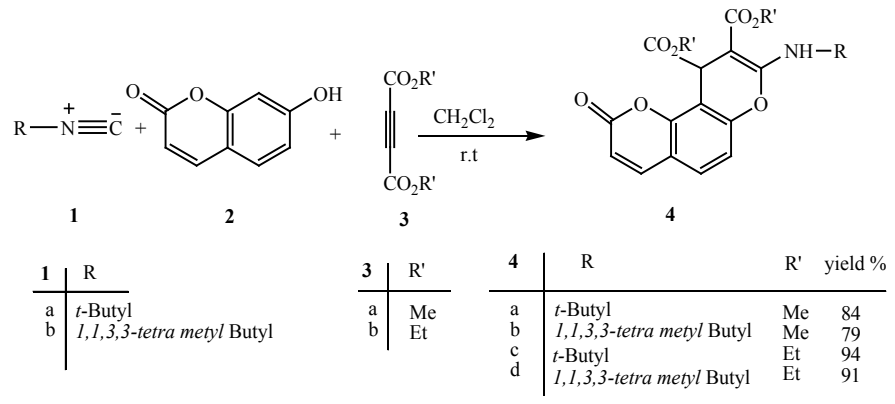

Scheme 1. 
On the basis of the well established chemistry of isocyanides [28-30] compound 4 result from nucleophilic addition of alkyl isocyanides to the acetylenic system and subsequent protonation of the 1:1 adduct by the $\mathrm{OH}$-acid. Then, the positively charged ion $\mathbf{6}$ is attacked by the anion of the $\mathrm{OH}$-acid to form ketenimine 7 (strong ketenimine absorption band at about $v=$ $2049 \mathrm{~cm}^{-1}$ was detected by IR spectroscopy during the reaction). Such an addition product may tautomerize to $\mathbf{8}$ and then cyclize, under the reaction conditions employed, to produce 4 . Direct addition to 6 leads to 1-aza-1,3-diene 5 (Scheme 3).

It seems that direct attack to $c$-hexyl isocyanide is more favorable due to weaker steric effect than tert-butyl and 1,1,3,3tetramethyl butyl isocyanides.

\section{Conclusion}

In conclusion, we have found an efficient synthetic method for the preparation of some $4 H$-chromenes and 1-azabuta-1, 3dienes. The present method carries the advantage that not only is the reaction performed under neutral conditions and without anhydrous conditions at room temperature, but also the starting materials and reagents can be mixed without any activation or modification.

\section{Experimental}

Elemental analyses for $\mathrm{C}, \mathrm{H}$, and $\mathrm{N}$ were performed using a Heraeus CHN-O-Rapid analyzer. IR spectra were measured on a Shimadzu IR-460 spectrometer. ${ }^{1} \mathrm{H}$ and ${ }^{13} \mathrm{C}$ NMR spectra were measured with a Bruker DRX-300 AVANCE instrument with $\mathrm{CDCl}_{3}$ as solvent at 300.1 and $75.5 \mathrm{MHz}$, respectively. Mass spectra were recorded on a Finnigan-Matt 8430 mass spectrometer operating at an ionization potential of $70 \mathrm{eV}$. Alkyl isocyanides, alkyl acetylenedicarboxylates and 7-hydroxycoumarin were obtained from Fluka (Buchs, Switzerland) and were used without further purification.

\section{Typical procedure for preparation of compounds 4 and 5}

To a magnetically stirred solution of 7-hydroxycoumarin $(2 \mathrm{mmol})$ and dimethyl acetylenedicarboxylate $(2 \mathrm{mmol})$ in $\mathrm{CH}_{2} \mathrm{Cl}_{2}(10 \mathrm{~mL})$ tert-butyl isocyanide $(2 \mathrm{mmol})$ was added dropwise at $-10{ }^{\circ} \mathrm{C}$ over $10 \mathrm{~min}$. The reaction mixture was then allowed to warm up to room temperature and stand for $24 \mathrm{~h}$. The solvent was removed under reduced pressure and the residue was separated by silica gel column chromatography (Merck 230-400 mesh) using (hexane: EtOAc, 5:1) as eluent.

Dimethyl 8-(tert-butylamino)-2-oxo-2H, 10H-pyrano[2,3f]chromene-9,10-dicarboxylate (4a). Yellow oil, yield: $0.65 \mathrm{~g}$ (84\%). IR (KBr): 3466, 1735, 1671, 1621, 1439, 1243, 1085 $\mathrm{cm}^{-1} .{ }^{1} \mathrm{H}$ NMR $\left(300 \mathrm{MHz}, \mathrm{CDCl}_{3}\right) \delta 8.76(1 \mathrm{H}, \mathrm{br} \mathrm{s}, \mathrm{NH}), 7.67$ $(1 \mathrm{H}, \mathrm{d}, J=9.6 \mathrm{~Hz}, \mathrm{CH}), 7.40(1 \mathrm{H}, \mathrm{d}, J=8.5 \mathrm{~Hz}, \mathrm{CH}), 7.03$ $(1 \mathrm{H}, \mathrm{d}, J=8.5 \mathrm{~Hz}, \mathrm{CH}), 6.36(1 \mathrm{H}, \mathrm{d}, J=9.5 \mathrm{~Hz}, \mathrm{CH}), 5.27$ $(1 \mathrm{H}, \mathrm{s}, \mathrm{CH}), 3.74\left(3 \mathrm{H}, \mathrm{s}, \mathrm{CH}_{3}-\mathrm{O}\right), 3.64\left(3 \mathrm{H}, 3, \mathrm{CH}_{3}-\mathrm{O}\right), 1.46$ $\left(9 \mathrm{H}, \mathrm{s}, 3 \mathrm{CH}_{3}\right) \cdot{ }^{13} \mathrm{C} \mathrm{NMR}\left(75.5 \mathrm{MHz}, \mathrm{CDCl}_{3}\right) \delta 172.9(\mathrm{C}), 169.6$ $(\mathrm{C}=\mathrm{O}), 161.8(\mathrm{C}=\mathrm{O}), 160.2(\mathrm{C}=\mathrm{O}), 152.4(\mathrm{C}), 151.8(\mathrm{C}), 143.2$ $(\mathrm{CH}), 127.6(\mathrm{CH}), 115.5(\mathrm{C}), 114.9(\mathrm{CH}), 112.7(\mathrm{CH}), 110.7$ (C), $72.1(\mathrm{C}), 52.7(\mathrm{C}-\mathrm{N}), 52.6\left(\mathrm{CH}_{3}-\mathrm{O}\right), 51.0\left(\mathrm{CH}_{3}-\mathrm{O}\right), 38.6$

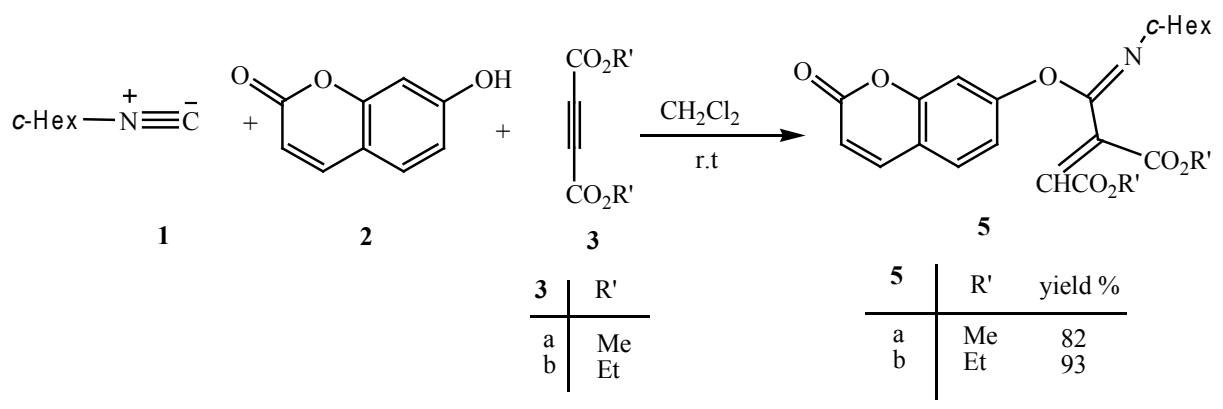

Scheme 2.

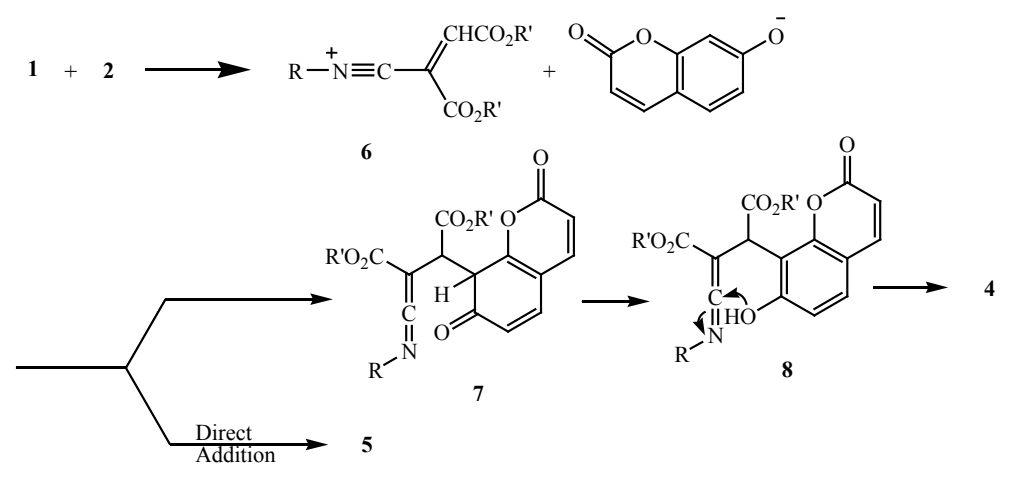

Scheme 3. 
$(\mathrm{CH}), 31.2\left(3 \mathrm{CH}_{3}\right)$. EI-MS $\mathrm{m} / z$ (rel.int.): $387\left[\mathrm{M}^{+}\right]$(13), 238 (43), 272 (54), 161 (72), 142 (80), 83 (78), 57 (100). Anal. calcd. for $4 \mathrm{a}, \mathrm{C}_{20} \mathrm{H}_{21} \mathrm{NO}_{7}: \mathrm{C}, 62.01 ; \mathrm{H}, 5.46 ; \mathrm{N}, 3.62$; found: C, 62.04; H, 5.45; N, 3.60 .

Dimethyl 8-(2,4,4-trimethylpentan-2-ylamino)-2-oxo-2H, 10H-pyrano[2,3-f]chromene-9,10-dicarboxylate (4b). Yellow oil, yield: $0.70 \mathrm{~g}(79 \%)$. IR (KBr): $3450,1738,1668,1623$, 1427, 1224, $1072 \mathrm{~cm}^{-1} .{ }^{1} \mathrm{H}$ NMR $\left(300 \mathrm{MHz}, \mathrm{CDCl}_{3}\right) \delta 8.83$ $(1 \mathrm{H}, \mathrm{br} \mathrm{s}, \mathrm{NH}), 7.67(1 \mathrm{H}, \mathrm{d}, J=9.6 \mathrm{~Hz}, \mathrm{CH}), 7.41(1 \mathrm{H}, \mathrm{d}, J$ $=8.5 \mathrm{~Hz}, \mathrm{CH}), 7.03(1 \mathrm{H}, \mathrm{d}, J=8.5 \mathrm{~Hz}, \mathrm{CH}), 6.37(1 \mathrm{H}, \mathrm{d}, J=$ $9.5 \mathrm{~Hz}, \mathrm{CH}), 5.29(1 \mathrm{H}, \mathrm{s}, \mathrm{CH}), 3.79\left(3 \mathrm{H}, \mathrm{s}, \mathrm{CH}_{3}-\mathrm{O}\right), 3.64(3 \mathrm{H}$, s, $\left.\mathrm{CH}_{3}-\mathrm{O}\right), 1.90\left(2 \mathrm{H}, \mathrm{s}, \mathrm{CH}_{2}\right), 1.53\left(3 \mathrm{H}, \mathrm{s}, \mathrm{CH}_{3}\right), 1.51(3 \mathrm{H}, \mathrm{s}$, $\left.\mathrm{CH}_{3}\right), 1.00\left(9 \mathrm{H}, \mathrm{s}, 3 \mathrm{CH}_{3}\right) \cdot{ }^{13} \mathrm{C} \mathrm{NMR}\left(75.5 \mathrm{MHz}, \mathrm{CDCl}_{3}\right) \delta$ 172.7(C), $169.5(\mathrm{C}=\mathrm{O}), 161.6(\mathrm{C}=\mathrm{O}), 160.1(\mathrm{C}=\mathrm{O}), 152.3(\mathrm{C})$, $151.7(\mathrm{C}), 143.1(\mathrm{CH}), 127.6(\mathrm{CH}), 115.4(\mathrm{C}), 115.0(\mathrm{CH})$, $112.6(\mathrm{CH}), 110.8(\mathrm{C}), 71.7(\mathrm{C}), 56.2(\mathrm{C}-\mathrm{N}), 55.1\left(\mathrm{CH}_{3}-\mathrm{O}\right)$, $53.4\left(\mathrm{CH}_{2}\right), 51.1\left(\mathrm{CH}_{3}-\mathrm{O}\right), 34.9(\mathrm{CH}), 31.7\left(\mathrm{CH}_{3}\right), 31.6(\mathrm{C})$, $31.4\left(3 \mathrm{CH}_{3}\right), 31.1\left(\mathrm{CH}_{3}\right)$. EI-MS $m / z$ (rel.int.): $443\left[\mathrm{M}^{+}\right](8)$, 383 (35), 269 (100), 241 (24), 57 (73), 41 (59). Anal. calcd. for 4b, $\mathrm{C}_{24} \mathrm{H}_{29} \mathrm{NO}_{7}$ : C, 65.00; $\mathrm{H}, 6.59$; N, 3.16; found: $\mathrm{C}, 65.02$; $\mathrm{H}, 6.58 ; \mathrm{N}, 3.14$.

Diethyl 8-(tert-butylamino)-2-oxo-2H, 10H-pyrano[2,3f]chromene-9,10-dicarboxylate (4c). Yellow oil, yield: $0.78 \mathrm{~g}$ (94\%). IR (KBr): 3439, 1738, 1667, 1624, 1440, 1218, 1076 $\mathrm{cm}^{-1} .{ }^{1} \mathrm{H}$ NMR $\left(300 \mathrm{MHz}, \mathrm{CDCl}_{3}\right) \delta 8.82(1 \mathrm{H}, \mathrm{br} \mathrm{s}, \mathrm{NH}), 7.67$ $(1 \mathrm{H}, \mathrm{d}, J=9.5 \mathrm{~Hz}, \mathrm{CH}), 7.40(1 \mathrm{H}, \mathrm{d}, J=8.5 \mathrm{~Hz}, \mathrm{CH}), 7.03$ $(1 \mathrm{H}, \mathrm{d}, J=8.5 \mathrm{~Hz}, \mathrm{CH}), 6.36(1 \mathrm{H}, \mathrm{d}, J=9.5 \mathrm{~Hz}, \mathrm{CH}), 5.24$ $(1 \mathrm{H}, \mathrm{s}, \mathrm{CH}), 4.28\left(2 \mathrm{H}, \mathrm{m}, \mathrm{CH}_{2}-\mathrm{O}\right), 4.10\left(2 \mathrm{H}, \mathrm{m}, \mathrm{CH}_{2}-\mathrm{O}\right), 1.47$ $\left(9 \mathrm{H}, \mathrm{s}, 3 \mathrm{CH}_{3}\right), 1.33\left(3 \mathrm{H}, \mathrm{t}, J=7.1 \mathrm{~Hz}, \mathrm{CH}_{3}\right), 1.21(3 \mathrm{H}, \mathrm{t}, J=$ $\left.7.1 \mathrm{~Hz}, \mathrm{CH}_{3}\right) \cdot{ }^{13} \mathrm{C} \mathrm{NMR}\left(75.5 \mathrm{MHz}, \mathrm{CDCl}_{3}\right) \delta 172.3(\mathrm{C}), 168.9$ $(\mathrm{C}=\mathrm{O}), 161.1(\mathrm{C}=\mathrm{O}), 159.8(\mathrm{C}=\mathrm{O}), 151.9(\mathrm{C}), 151.5(\mathrm{C}), 142.8$ $(\mathrm{CH}), 127.1(\mathrm{CH}), 114.9(\mathrm{C}), 114.5(\mathrm{CH}), 112.3(\mathrm{CH}), 110.3$ (C), $71.7(\mathrm{C}), 60.8\left(\mathrm{CH}_{2}-\mathrm{O}\right), 59.2\left(\mathrm{CH}_{2}-\mathrm{O}\right), 52.2(\mathrm{C}-\mathrm{N}), 38.3$ (CH), $30.2\left(3 \mathrm{CH}_{3}\right), 14.3\left(\mathrm{CH}_{3}\right), 13.7\left(\mathrm{CH}_{3}\right)$. EI-MS $\mathrm{m} / z$ (rel. int.): $415\left[\mathrm{M}^{+}\right]$(12), 337 (100), 292 (43), 255 (66), 223 (84), 57 (96). Anal. calcd. for $4 \mathbf{c}, \mathrm{C}_{22} \mathrm{H}_{25} \mathrm{NO}_{7}: \mathrm{C}, 63.60 ; \mathrm{H}, 6.07 ; \mathrm{N}$, 3.37; O, 26.96; found: C, 63.62; H, 6.06; N, 3.35.

Diethyl 8-(2,4,4-trimethylpentan-2-ylamino)-2-oxo-2H, 10H-pyrano[2,3-f]chromene-9,10-dicarboxylate (4d). Yellow oil, yield: $0.86 \mathrm{~g}(91 \%)$. IR (KBr): 3426, 1732, 1668, 1621, $1428,1215,1071 \mathrm{~cm}^{-1} .{ }^{1} \mathrm{H}$ NMR $\left(300 \mathrm{MHz}, \mathrm{CDCl}_{3}\right) \delta 8.87(1 \mathrm{H}$, br s, NH), $7.68(1 \mathrm{H}, \mathrm{d}, J=9.5 \mathrm{~Hz}, \mathrm{CH}), 7.41(1 \mathrm{H}, \mathrm{d}, J=8.5$ $\mathrm{Hz}, \mathrm{CH}), 7.02(1 \mathrm{H}, \mathrm{d}, J=8.5 \mathrm{~Hz}, \mathrm{CH}), 6.34(1 \mathrm{H}, \mathrm{d}, J=9.5 \mathrm{~Hz}$, $\mathrm{CH}), 5.24(1 \mathrm{H}, \mathrm{s}, \mathrm{CH}), 4.15\left(2 \mathrm{H}, \mathrm{m}, \mathrm{CH}_{2}-\mathrm{O}\right), 4.07\left(2 \mathrm{H}, \mathrm{m}, \mathrm{CH}_{2^{-}}\right.$ O), $1.80\left(2 \mathrm{H}, \mathrm{s}, \mathrm{CH}_{2}\right), 1.51\left(3 \mathrm{H}, \mathrm{s}, \mathrm{CH}_{3}\right), 1.49\left(3 \mathrm{H}, \mathrm{s}, \mathrm{CH}_{3}\right)$, $1.31\left(3 \mathrm{H}, \mathrm{t}, J=7.1 \mathrm{~Hz}, \mathrm{CH}_{3}\right), 1.21\left(3 \mathrm{H}, \mathrm{t}, J=7.1 \mathrm{~Hz}, \mathrm{CH}_{3}\right)$, $0.98\left(9 \mathrm{H}, \mathrm{s}, 3 \mathrm{CH}_{3}\right) .{ }^{13} \mathrm{C} \mathrm{NMR}\left(75.5 \mathrm{MHz}, \mathrm{CDCl}_{3}\right) \delta 172.6(\mathrm{C})$, $169.3(\mathrm{C}=\mathrm{O}), 161.4(\mathrm{C}=\mathrm{O}), 160.2(\mathrm{C}=\mathrm{O}), 152.3(\mathrm{C}), 151.9(\mathrm{C})$, $143.2(\mathrm{CH}), 127.6(\mathrm{CH}), 115.4(\mathrm{C}), 114.9(\mathrm{CH}), 112.6(\mathrm{CH})$, $110.8(\mathrm{C}), 71.8(\mathrm{C}), 61.2\left(\mathrm{CH}_{2}-\mathrm{O}\right), 59.5\left(\mathrm{CH}_{2}-\mathrm{O}\right), 56.2(\mathrm{C}-\mathrm{N})$, $53.5\left(\mathrm{CH}_{2}\right), 35.3(\mathrm{CH}), 31.7\left(\mathrm{CH}_{3}\right), 31.6(\mathrm{C}), 31.4\left(3 \mathrm{CH}_{3}\right), 31.2$ $\left(\mathrm{CH}_{3}\right), 14.7\left(\mathrm{CH}_{3}\right), 14.1\left(\mathrm{CH}_{3}\right)$. EI-MS $\mathrm{m} / z$ (rel.int.): $471\left[\mathrm{M}^{+}\right]$ (7), 398 (39), 179 (60), 369 (46), 313 (53), 286 (100), 240 (61), 57 (96). Anal. calcd. for $4 \mathbf{d}, \mathrm{C}_{26} \mathrm{H}_{33} \mathrm{NO}_{7}: \mathrm{C}, 66.22 ; \mathrm{H}, 7.05 ; \mathrm{N}$, 2.97; found; C, 66.25; H, 7.04; N, 2.95 .
Dimethyl 2-((1Z)-(2-oxo-2H-chromen-7-yloxy)(cyclohexyl imino)methyl)but-2-enedioate (5a). Yellow oil, yield: $0.68 \mathrm{~g}$ (82\%). IR (KBr): 1739, 1682, 1670, 1616, 1230, $1120 \mathrm{~cm}^{-1}$. ${ }^{1} \mathrm{H}$ NMR $\left(300 \mathrm{MHz}, \mathrm{CDCl}_{3}\right) \delta 7.68(1 \mathrm{H}, \mathrm{d}, J=9.5 \mathrm{~Hz}, \mathrm{CH})$, $7.42(1 \mathrm{H}, \mathrm{d}, J=8.5 \mathrm{~Hz}, \mathrm{CH}), 7.35(1 \mathrm{H}, \mathrm{d}, J=2.0 \mathrm{~Hz}, \mathrm{CH}), 7.20$ $(1 \mathrm{H}, \mathrm{dd}, J=8.5,2.1 \mathrm{~Hz}, \mathrm{CH}), 7.10(1 \mathrm{H}, \mathrm{s}, \mathrm{CH}), 6.34(1 \mathrm{H}, \mathrm{d}$, $J=9.5 \mathrm{~Hz}, \mathrm{CH}), 3.91\left(3 \mathrm{H}, \mathrm{s}, \mathrm{CH}_{3}-\mathrm{O}\right), 3.82\left(3 \mathrm{H}, \mathrm{s}, \mathrm{CH}_{3}-\mathrm{O}\right)$, $2.99(1 \mathrm{H}, \mathrm{m}, \mathrm{CH}), 1.18-1.66\left(10 \mathrm{H}, \mathrm{m}, 5 \mathrm{CH}_{2}\right) .{ }^{13} \mathrm{C}$ NMR $(75.5$ $\left.\mathrm{MHz}, \mathrm{CDCl}_{3}\right) \delta 163.4(\mathrm{C}=\mathrm{O}), 163.0(\mathrm{C}=\mathrm{O}), 160.6(\mathrm{C}=\mathrm{O}), 155.6$ (C), $154.3(\mathrm{C}), 150.7(\mathrm{C}), 142.9(\mathrm{CH}), 136.3(\mathrm{CH}), 131.8(\mathrm{CH})$, 127.6 (CH), $118.2(\mathrm{CH}), 115.2(\mathrm{C}), 114.6(\mathrm{CH}), 109.9(\mathrm{C}), 52.5$ $\left(\mathrm{CH}_{3}-\mathrm{O}\right), 51.1\left(\mathrm{CH}_{3}-\mathrm{O}\right), 49.9(\mathrm{CH}), 38.3(\mathrm{CH}), 33.9\left(\mathrm{CH}_{2}\right) 33.6$ $\left(\mathrm{CH}_{2}\right), 25.5\left(\mathrm{CH}_{2}\right), 24.6\left(\mathrm{CH}_{2}\right), 24.5\left(\mathrm{CH}_{2}\right)$. EI-MS $\mathrm{m} / z(\mathrm{rel}$. int.): $413\left[\mathrm{M}^{+}\right]$(21), 337 (100), 292 (23), 255 (66), 223 (84), 83 (74), 55 (27). Anal. calcd. for $5 \mathbf{a}, \mathrm{C}_{22} \mathrm{H}_{23} \mathrm{NO}_{7}: \mathrm{C}, 63.91 ; \mathrm{H}$, 5.61; N, 3.39; found: C, 63.93; H, 5.62; N, 3.35.

Diethyl 2-(1Z)-(2-oxo-2H-chromen-7-yloxy)(cyclohexyli mino)methyl)but-2-enedioate (5b). Yellow oil, yield: $0.92 \mathrm{~g}$ (93\%). IR (KBr): 1729, 1682, 1655, 1613, 1255, $1118 \mathrm{~cm}^{-1} .{ }^{1} \mathrm{H}$ NMR $\left(300 \mathrm{MHz}, \mathrm{CDCl}_{3}\right) \delta 7.67(1 \mathrm{H}, \mathrm{d}, J=9.5 \mathrm{~Hz}, \mathrm{CH}), 7.42$ $(1 \mathrm{H}, \mathrm{d}, J=8.5 \mathrm{~Hz}, \mathrm{CH}), 7.34(1 \mathrm{H}, \mathrm{d}, J=2.0 \mathrm{~Hz}, \mathrm{CH}), 7.19(1 \mathrm{H}$, dd, $J=8.4,2.1 \mathrm{~Hz}, \mathrm{CH}), 7.08(1 \mathrm{H}, \mathrm{s}, \mathrm{CH}), 6.33(1 \mathrm{H}, \mathrm{d}, J=9.5$ $\mathrm{Hz}, \mathrm{CH}), 4.34\left(2 \mathrm{H}, \mathrm{q}, J=7.1 \mathrm{~Hz}, \mathrm{CH}_{2}-\mathrm{O}\right), 4.28(2 \mathrm{H}, \mathrm{q}, J=7.1$ $\left.\mathrm{Hz}, \mathrm{CH}_{2}-\mathrm{O}\right), 3.00(1 \mathrm{H}, \mathrm{m}, \mathrm{CH}), 11.34\left(3 \mathrm{H}, \mathrm{t}, J=7.1 \mathrm{~Hz}, \mathrm{CH}_{3}\right)$, $1.28\left(3 \mathrm{H}, \mathrm{t}, J=7.1 \mathrm{~Hz}, \mathrm{CH}_{3}\right), 17-1.85\left(10 \mathrm{H}, \mathrm{m}, 5 \mathrm{CH}_{2}\right) \cdot{ }^{13} \mathrm{C}$ NMR (75.5 MHz, $\left.\mathrm{CDCl}_{3}\right) \delta 163.4(\mathrm{C}=\mathrm{O}), 163.0(\mathrm{C}=\mathrm{O}), 161.6$ $(\mathrm{C}=\mathrm{O}), 155.6(\mathrm{C}), 154.3(\mathrm{C}), 150.3(\mathrm{C}), 142.9(\mathrm{C}), 136.3(\mathrm{C})$, $131.4(\mathrm{CH}), 127.8(\mathrm{CH}), 118.2(\mathrm{CH}), 115.2(\mathrm{CH}), 114.6(\mathrm{CH})$, $109.9(\mathrm{CH}), 71.3(\mathrm{C}), 61.1\left(\mathrm{CH}_{2}-\mathrm{O}\right), 59.4\left(\mathrm{CH}_{2}-\mathrm{O}\right), 49.9(\mathrm{CH})$, $38.5(\mathrm{CH}), 33.9\left(\mathrm{CH}_{2}\right), 33.6\left(\mathrm{CH}_{2}\right), 25.5\left(\mathrm{CH}_{2}\right), 24.5\left(2 \mathrm{CH}_{2}\right)$, $14.7\left(\mathrm{CH}_{3}\right), 14.1\left(\mathrm{CH}_{3}\right)$. EI-MS $m / z$ (rel.int.): $441\left[\mathrm{M}^{+}\right](17)$, 339 (85), 285 (14), 255 (66), 223 (84), 83 (100). Anal. calcd. for $5 \mathbf{b}, \mathrm{C}_{24} \mathrm{H}_{27} \mathrm{NO}_{7}$ : C, 65.29; $\mathrm{H}, 6.16$; N, 3.17; found: C, 65.28; $\mathrm{H}, 6.17 ; \mathrm{N}, 3.18$.

\section{References}

1. Hoffmann, P.; Gokel, G.; Marquarding, D.; Ugi, I. in Isonitrile Chemistry, Ugi, I., Ed., Academic Press: New York, 1971.

2. (a) Ugi, I. Pure Appl. Chem. 2001, 73, 187-191. (b) Ugi, I.; Werner, B.; Dömling, A. Molecules 2003, 8, 53-66. (c) Hulme, C.; Gore, V. Curr. Med. Chem. 2003, 10, 51-80.

3. Dömling, A.; Ugi, I. Angew. Chem., Int. Ed. 2000, 39, 31683210.

4. Dömling, A. Chem. Rev. 2006, 106, 17-89.

5. Dömling, A.; Wang, W.; Wang, K. Chem. Rev. 2012, 112, 30833135.

6. Graaff, C.; Ruijter, E.; Orru, R. V. Chem. Soc. Rev. 2012, 41, 3969-4009.

7. Nair, V.; Vinod, A. U.; Abhilash, N.; Menon, R. S.; Santhi, V.; Varma, R. L.; Viji, S.; Mathewa, S.; Srinivas, R. Tetrahedron 2003, 59, 10279-10286.

8. Nair, V.; Vinod, A. U.; Ramesh, R.; Menon, R. S.; Varma, L.; Mathew, S.; Chiaroni, A. Heterocycles 2002, 58, 147-151.

9. Adib, M.; Sayahi, M. H.; Rahbari, S. Tetrahedron Lett. 2005, 46, 6545-6547.

10. Beaudegnies, R.; Ghosez, L. Tetrahedron: Asymmetry 1994, 5, 557-560. 
11. Yu. N.; Aramini, J. M.; Germann, M. W.; Huang, Z. Tetrahedron Lett. 2000, 41, 6993-6996.

12. Bloxam, J.; Dell, C. P.; Smith, C. W. Heterocycles 1994, 38, 399408.

13. Yavari, I.; Djahaniani, H.; Nasiri, F. Tetrahedron 2003, 59, 94099412.

14. Yavari, I.; Anary-Abbasinejad, M.; Alizadeh, A.; Hossaini, Z. Tetrahedron 2003, 59, 1289-1292.

15. Yavari, I.; Djahaniani, H.; Nasiri, F. Synthesis 2004, 679-682.

16. Yavari, I.; Djahaniani, H.; Nasiri, F. Mendeleev Commun. 2004, 214-216.

17. Teimouri, M. B.; Bazhrang, R.; Eslamimanesh, V.; Nouri, A. Tetrahedron 2006, 62, 3016-3020.

18. Yavari, I.; Hossaini, Z.; Sabbaghan, M. Monatsh. Chem. 2007, 138, 107-110.

19. Sarma, R.; Sarmah, M. M.; Lekhok, K. C.; Prajapati, D. Synlett 2010, 19, 2847-2852.

20. Mohtat, B.; Djahaniani, H.; Yavari, I.; Dehbalaei, M.G.; Jam, S.A. Chin. Chem. Lett. 2011, 22, 771-773.
21. Mohtat, B.; Djahaniani, H.; Khorrami, R.; Mashayekhi, S.; Yavari, I. Synth. Commun. 2011, 41, 784-791.

22. Mohtat, B.; Djahaniani, H.; Yavari, I.; Naderi, K.; J. Serb. Chem. Soc. 2011, 76, 13-20.

23. Mohtat, B.; Rezazadeh, S.; Matinfar, M.; Arabzadeh, V.; Djahaniani, H.; Hossaini, Z. Lett. Org. Chem. 2012, 9, 150-153.

24. Mohtat, B.; Najafi Azar, Z.; Nahavandian, S.; Djahaniani, H.; Ahmadi, A. J. Mex. Chem. Soc. 2011, 55, 194-196.

25. Yavari, I.; Zare, H.; Mohtat, B. Mol. Divers. 2006, 10, 247-250.

26. Yavari, I.; Zare, H.; Mohtat, B. J. Chem. Res. (S). 2007, 152-154.

27. Pretsch, E.; Buhlmann, P.; Badertscher, M. Structure Determination of Organic Compounds Springer-Verlag Berlin Heidelberg, 2009.

28. Ugi, I. Angew. Chem., Int. Ed. Engl. 1982, 21, 810-819.

29. Walborsky, H. M.; Periasamy, M. P. in: The Chemistry of Functional Groups, Chapter 20, Patai, S.; Rappoport, Z. Eds., Wiley, New York, 1983, 835.

30. Marcaccini, S.; Torroba, T. Org. Prep. Proced. Int. 1993, 25, 141208 